\title{
Low-cost home experiments and demonstrations in optics
}

P. Mejías, R. Martínez-Herrero, J. Serna, G. Piquero

P. M. Mejías, R. Martínez-Herrero, J. Serna, G. Piquero, "Low-cost home experiments and demonstrations in optics," Proc. SPIE 9664, Ninth International Topical Meeting on Education and Training in Optics and Photonics, 966426 (24 October 2005); doi: 10.1117/12.2207567

Event: Ninth International Topical Meeting on Education and Training in Optics and Photonics, 2005, Marseille, France 
Ref ETOP016

Low-cost home experiments and demonstrations in Optics

P. M. Mejías, R. Martínez-Herrero, J. Serna, G. Piquero

Departamento de Óptica, Facultad CC. Físicas, Universidad Complutense, 28040

Madrid, Spain, pmmejias@fis.ucm.es

\section{Abstract}

More than 60 demonstrations and basic experiments in Optics have been compiled. They can be carried out by secondary and university students in the classroom or at home, and have been conceived considering low cost and easy-to-get materials. The goal is to offer didactic resources, showing that Optics can be taught in an attractive and amusing way. The experiments try to stimulate scientific curiosity, and generate interest in the observation of our physical world. The work could be collected as a book, where each demonstration would be contained in one or two pages, including a title, a list of the required materials and a concise explanation about what to do and observe. Associated with the experimental content, we propose a web page, namely, http://www.ucm.es/info/expoptic, that accepts experiments sent by anyone interested in Optics, which can be used as a forum to interchange information on this educational topic.

\section{Summary}

In this work we have prepared more than 60 low cost demonstrations and experiments in Optics. They can be used in the classroom or at home, and have been designed by handling inexpensive and easy to find equipment and materials. Our goals are

- To present a pleasant and fun approach to learning Optics

- To offer didactical resources for the classroom

- To stimulate the scientific curiosity of students in this field

- To create interest in the observation of the surrounding physical world

The compilation is addressed in first term to students in the initial university courses. During these years, university programs usually include the theoretical foundations that allow a rigorous and detailed understanding of each experiment. Nonetheless, most experiences are also adequate for secondary school level. In this case, teacher supervision could be important, especially in order to provide the appropriate background. In any case, the collection is also directed to anyone interested in Optics in a wide sense.

Due to their simplicity, the collected experiments can be used to support Optics lessons. They could also become specially helpful because, on the one hand, classical laboratories cannot cover such a wide range of optical phenomena, and, on the other hand, theoretical and laboratory classes could not be simultaneous.

Demonstrations have been classified in two big sections, Geometrical Optics and Wave Optics, following the standard division used in Optics courses. In the Geometrical Optics 
section there are experiments on the straight line propagation and independence of light rays, reflection law, total internal reflection, Snell's law, propagation in inhomogeneous media, lenses, mirrors, refractive index, stops, aberrations, image forming instruments and photometry. Within the Wave Optics section we have included experiments on dispersion, absorption, scattering, polarization, birefringence, dichroism, optical activity, interference and diffraction.

Each experiment description includes a title followed by a list of required materials and a brief explanation on how to proceed. The detailed theoretical explanation can be found in the recommended bibliography, and it depends on the educational level. Appropriate demonstrations for the classroom are pointed out. We also explain how to buy, prepare or use some specific materials.

Associated with the experimental work, we propose a web page (preliminary version in Spanish: http://www.ucm.es/info/expoptic), which has been conceived as an exchange forum for teachers, students and anyone interested in low cost experiments in optics. Any person can send suggestions and experiments descriptions adequate to be uploaded to the web page. Experiments can be accessed by subject, materials and suitability for the classroom.

\section{References}

1.P. M. Mejías, R. Martínez-Herrero, Enseñanza de las Ciencias, special issue, 263 (1987).

2. P. M. Mejías, R. Martínez-Herrero, R. Cid, Enseñanza de las Ciencias, special issue, 264 (1989).

3. P. M. Mejías, in Apparatus for Teaching Physics, K. C. Mamola (ed.), American Association of Physics Teachers, 207 (1998).

4. J. Serna, G. Piquero, Eur. J. Phys., vol. 23, 465 (2002).

5. J. Serna, G. Piquero, II Feria Madrid por la Ciencia, 162. Comunidad de Madrid/Ediciones SM, 2001.

6. R. Martínez-Herrero, P. M. Mejías, J. Serna, V Feria Madrid por la Ciencia, 228. Comunidad de Madrid/Ediciones SM, 2004. 